\title{
MAKING A HOLE IN THE SPACE
}

\author{
KAZIMIERZ GOEBEL AND JACEK WOŚKO
}

(Communicated by Paul S. Muhly)

\begin{abstract}
The paper provides a simple recipe for a construction of homeomorphisms "removing" convex bodies from nonreflexive Banach spaces.
\end{abstract}

Let $X$ be a Banach space. A set $K \subset X$ is said to be removable (or deleting) from $X$ if there exists a homeomorphism $T$ mapping $X$ onto $X \backslash K$. We say also that $T$ removes $K$ from $X$.

The study of removable sets was initiated by Klee [1] with the observation that if $X$ is of infinite dimension then whole space is homeomorphic to the punctured space $X \backslash\{0\}$. Detailed discussion of the theory can be found in Bessaga and Pełczyński's book [2].

For the newcomer to the theory some facts as mentioned above may seem surprising, especially that the methods used in proofs are often "nonconstructive."

Below we present a hopefully unknown recipe for a construction of homeomorphisms removing from the spaces a closed and bounded convex body, a ball or a point. Our construction works, however, only in nonreflexive Banach spaces.

Let $X$ be nonreflexive and let $B$ and $S$ stand for the closed unit ball and the unit sphere, respectively.

Since $X$ is nonreflexive, there exists a linear functional $f \in X^{*}$ such that $\|f\|=1$ which does not achieve its maximum on $B$, which means that $|f(x)|<$ $\|x\|$ for all $x \neq 0$.

Consider the function $\phi$ defined on $X$ by

$$
\begin{aligned}
\phi(x) & =1+f(x)+\max [0,2(\|x\|-1)] \\
& = \begin{cases}1+f(x), & \text { for } x \in B, \\
2 \cdot\|x\|-1+f(x), & \text { for } x \notin B,\end{cases}
\end{aligned}
$$

and observe that $\phi$ is continuous (lipschitzian) convex and even affine when restricted to $B$. Moreover $\lim _{\|x\| \rightarrow+\infty} \phi(x)=+\infty$ while

$$
\inf [\phi(x): x \in X]=\inf [\phi(x): x \in B]=0 .
$$

Observe also that this infimum is not taken and $\phi(B)=\phi(S)=(0,2)$.

Received by the editors August 5, 1990 .

1980 Mathematics Subject Classification (1985 Revision). Primary 47H99. 
Select a sequence of points $\left\{x_{n}\right\}, n=0,1,2, \ldots$ on the unit sphere $S$ such that $\phi\left(x_{n}\right)=2^{-n}$.

Form a curve $\gamma$ by joining consecutive points by segments and complete it by joining $x_{0}$ with infinity by a radial ray.

Observe that since $\phi$ is affine on $B$ this curve can be parametrized, $\gamma=\gamma(t)$, by $t \in(0, \infty)$ in such a way that $\phi(\gamma(t))=t$.

To end the preparation, for any $a \geq 0$ denote:

$$
K_{a}=[x: \phi(x) \leq a]
$$

and notice that for $a>0$ each $K_{a}$ is closed convex subset of $X$ having nonempty interior, while

$$
K_{0}=\bigcap_{a>0} K_{a}=\varnothing .
$$

Now we can formulate our recipe.

Fix a number $a \geq 0$. Take any $x \in X$. Calculate $\phi(x)$. Find the point $y$ on $\gamma$ such that $\phi(y)=\frac{1}{2} \phi(x)$ (simply take $y=\gamma\left(\frac{1}{2} \phi(x)\right)$ ). Obviously $y \neq x$. Follow the ray beginning at $x$ and passing through $y$ as far as you reach point $z$ such that $\phi(z)=\phi(x)+a$ (there is only one such point).

Define $T_{a}(x)=z$.

Observe that $T_{a}$ maps $X$ onto $X \backslash K_{a}$ and is invertible. It is only a technicality to prove that $T_{a}$ and $T_{a}^{-1}$ are continuous (even locally lipschitzian).

Thus $T_{a}$ removes $K_{a}$ from $X$ and for $a>0$ the hole mentioned in the title has been made. To make the hole ball shaped observe that $B \subset K_{2}$ and define

$$
T_{B}(x)=p\left(T_{2}(x)\right) T_{2}(x) /\left\|T_{2}(x)\right\|,
$$

where $p()$ denotes the Minkowski functional for $K_{2}$.

$T_{B}$ removes the unit ball from $X$, finally

$$
T(x)=\left(1-\frac{T_{2}(x)}{\left\|T_{2}(x)\right\|}\right) T_{B}(x)
$$

removes just one point, the origin.

Finally let us consider the case $a=0 . T_{0}$ does not remove anything from $X$ but it shows another "exotic" behavior. $T_{0}$ is self-invertible which means $T_{0}^{2}=I$ and at the same time $T_{0}$ does not have a fixed point $\left(x \neq T_{0}(x)\right) . T_{0}$ is only locally lipschitzian. The problem of whether there exists a uniformly continuous mapping $T$ having the above property was raised by Koter [3] and is still unsolved.

\section{REFERENCES}

1. V. Klee, Two topological properties of topological linear spaces, Israel J. Math. 4 (1964), 211-220.

2. Cz. Bessaga and A. Pełczyński, Selected topics in infinite-dimensional topology, Polish Scientific Publishers, 1975.

3. M. Koter, Fixed points of lipschitzian 2-rotative mappings, Anal. Funz. Appl. Ser. VI, V-C, (1986). 